\title{
Microelectrode-guided posteroventral pallidotomy for treatment of Parkinson's disease: postoperative magnetic resonance imaging analysis
}

Joachim K. Krauss, M.D., J. Michael Desaloms, M.D., Eugene C. Lai, M.D., Ph.D., David E. King, M.D., Joseph Jankovic, M.D., and Robert G. Grossman, M.D.

Departments of Neurosurgery, Neurology, and Neuroradiology, Baylor College of Medicine, Houston, Texas

The authors report the postoperative magnetic resonance (MR) imaging findings in 36 patients with advanced Parkinson's disease who underwent unilateral microelectrode-guided posteroventral pallidotomy. The lesions were placed within $1 \mathrm{~mm}$ of the ventral border of the globus pallidus internus (GPi) to include pallidothalamic outflow pathways. Sequential MR studies were obtained within 1 to 3 days postoperatively and at 6-month follow-up examination. Thirty-four (94\%) of the 36 patients enjoyed sustained moderate or marked improvement of their parkinsonian symptoms 6 months postoperatively. Transient side effects occurred in five patients (14\%), but there were no persistent complications. The pallidal radiofrequency lesions were prolate spheroid-shaped and were composed of three concentric zones in the early postoperative studies. The mean volume of the middle zone, corresponding to the area of hemorrhagic coagulation necrosis, was $44.4+/-17.6 \mathrm{~mm}^{3}$; the mean lesion volume as defined by the outer zone, corresponding to perilesional edema, was $262.2+/-111.6 \mathrm{~mm}^{3}$. Additional edema spreading to the internal capsule was noted in 32 of 34 cases and to the optic tract in 11 of 34 cases. In two patients small ischemic infarctions involving the corona radiata were found, and in one a venous infarction was detected. Ischemic infarction resulted in mild transient Broca's aphasia in one patient, but there was no detectable neurological deficit in the other two. The mean volume of late-phase (6 months) lesions was $22+/-28.8 \mathrm{~mm}^{3}$. In three patients no lesion was identified despite sustained clinical improvement. The lesion was located in the posteroventral GPi in all cases except in one patient in whom it was confined to the GP externus (GPe). This 49-year-old woman did not experience sustained benefit. The authors found no consistent correlations between lesion size and location and clinical outcome as measured by a global outcome score, the Unified Parkinson's Disease Rating Scale motor, activities of daily living, and bradykinesia "off" scores or rating of dyskinesias. Lesioning of pallidal and subpallidal pathways may contribute to the sustained clinical benefit in this series. Magnetic resonance imaging analysis showed that intraoperative microelectrode recording facilitated accurate placement of the lesion in this critical area.

Key Words * pallidotomy * Parkinson's disease * stereotaxy * magnetic resonance imaging

The surgical treatment of Parkinson's disease (PD) has witnessed a renaissance within the past few years. 
The refinement of techniques in neurosurgery, neuroimaging, and neurophysiology, the shortcomings of chronic levodopa therapy, and better understanding of the functional organization of the basal ganglia and the pathophysiology of PD have stimulated renewed interest in surgical procedures.[23] In particular, pallidotomy has attracted widespread attention and has rapidly been adopted by a number of centers.[11]

Pallidotomy was a common procedure for treatment of PD and postencephalitic parkinsonism in the 1950s.[33,36] However, after thalamotomy was introduced by Hassler and Riechert in 1952,[15] it gradually replaced pallidotomy in the late 1950s and early 1960s for treatment of parkinsonian symptoms, mainly because it more consistently relieved tremor. With large lesions in the posteroventral globus pallidus internus (GPi) and GP externus (GPe), Leksell and coworkers[39] observed more beneficial results for tremor, as well as improvement of bradykinesia, which they described as proportional to the decrease of rigidity. Most neurologists and neurosurgeons during that time would not have considered bradykinesia a symptom that could be ameliorated by pallidal lesioning.[14] Pallidotomy was essentially abandoned in the 1960s. It was not until the early 1990s, when Laitinen and coworkers[26,27] reported their results, that pallidotomy reemerged as an effective treatment for PD. Although this procedure is now performed routinely in several centers, relatively little information on its benefits and drawbacks is available in the peer-reviewed literature.[2,10,18,32] Unilateral microelectrode-guided pallidotomy is now accepted as a valid option for treatment of medically intractable levodopa-induced dyskinesias.[11] It may also be effective for bradykinesia, rigidity, tremor, postural instability, and gait disturbance. The procedure is associated with low morbidity, and symptomatic and functional improvement is achieved in the majority of patients. $[30,38]$ Many questions, however, remain to be answered.[20,35] Few data are available on postoperative magnetic resonance (MR) imaging.[8,29] It is unclear where the pallidal lesion should ideally be located, and what size it should have.[17,21,26,31]

In this prospective study we describe early and late (6-month) postoperative MR findings in a total of 36 patients who underwent unilateral microelectrode-guided posteroventral GPi pallidotomy. We also investigate the impact of lesion size and location on clinical outcome.

\section{CLINICAL MATERIAL AND METHODS}

\section{Patient Population}

This study is based on the first 36 of 38 consecutive patients who underwent unilateral posteroventral GPi pallidotomy for treatment of disabling bradykinesia and levodopa-induced dyskinesias. Two patients in whom no assessment at 6 months or later was available were excluded from this analysis. The mean age of the 36 patients was 60 years (range 40-75 years) at the time of the operation. There were 18 women and 18 men. All patients were diagnosed with idiopathic PD according to clinical criteria.[19,37] All patients were responsive to levodopa therapy, but their parkinsonian symptoms were insufficiently controlled and nearly all experienced both levodopa-induced dyskinesias and motor fluctuations. Exclusion criteria were features that suggested other parkinsonian syndromes, Hoehn and Yahr Stage V even when "on" medications, major depression, severe dementia, alcohol or drug abuse, the presence of other significant medical diseases, and evidence of marked vascular encephalopathy or severe cerebral atrophy on preoperative MR imaging. The upper age limit for patients was 75 years.

For this study, clinical outcome at the 6-month follow-up examination was analyzed. Formal assessment at 6 months was available for 34 patients. In two patients the 9-month clinical follow-up examination, which did not differ from their 3-month examination, was used. 


\section{Clinical Assessment and Follow-Up Examination}

Patients were evaluated using a modified Core Assessment Program for Intracerebral Transplantatons (CAPIT) protocol[28] and additional examinations. The standardized test batteries included: the Unified Parkinson's Disease Rating Scale (UPDRS); Hoehn and Yahr staging; movement and reaction times; Schwab and England scores; and neuropsychological examinations. The UPDRS evaluations were performed in the practically defined "off" state, 12 hours after the last levodopa dosage, and during the best "on" state. Subitems of the UPDRS were videotaped in both the "on" and "off" states. Clinical assessments were made preoperatively, 2 weeks after the operation, 3 months after the operation, and at subsequent 3-month intervals. All formal evaluations were performed by the neurological team members. In general, patients were maintained on the same or a similar levodopa regimen as preoperatively. Only minor adjustments were made when necessary.

The global outcome was rated on a 5-point scale by both the patient and the evaluating neurologist as: 0 , worse; 1 , unchanged; 2 , slightly improved; 3 , moderately improved; and 4, markedly improved. Selected items used for the analysis in the present study included the UPDRS motor "off" score, the UPDRS Activities of Daily Living "off" score, a UPDRS "off" subscore for appendicular bradykinesia (scores 23-26) contralateral to the side of surgery, and the percentage of awake time with dyskinesias. Levodopa-induced dyskinesias contralateral to the side of surgery were rated as: 0 , more or less unchanged; 1, moderately or markedly improved; and 2, abolished or almost completely abolished.

\section{Surgical Procedure}

The stereotactic frame was affixed to the patient's head and local anesthetic was applied. Propofol was used in the first few patients, but was later abandoned because it interfered with parkinsonian symptoms and elicited abnormal movements in individual patients.[22] After fixation of the Leksell G-frame (Elekta Instruments, Atlanta, GA) contiguous 1-mm axial scans through the third ventricle were obtained on a helical computerized tomography (CT) scanner (General Electric Medical Systems, Milwaukee, WI). The scans were transferred to a workstation, and the anterior commissure (AC) and posterior commissure (PC) were identified on axial views and on coordinated simultaneous, reformatted sagittal views. A tentative target in the posteroventral GPi was chosen 19 to $21 \mathrm{~mm}$ lateral to the midline, 4 to 5 $\mathrm{mm}$ below the AC-PC line, and 2 to $3 \mathrm{~mm}$ anterior to the midcommissural point. A misalignment correction algorithm was applied to correct for deviation of the AC-PC line in relation to the frame. The frame coordinates of the preliminary target were retranslated to CT coordinates and checked on interactive axial, sagittal, and coronal CT images for their proximity to the choroidal fissure and the internal capsule. The patient's hair was shaved at the site of the incison, and local anesthetic was injected. A burr hole was made 7 to $9 \mathrm{~cm}$ above the orbital rim and $2 \mathrm{~cm}$ lateral to the midline. The dura was coagulated and incised. The arachnoid was opened at the crown of a gyrus, carefully preserving small cortical arteries and bridging veins.

Microelectrode trajectories were planned at an angle of 40 to 455 above the AC-PC line and parallel to the midsagittal line. The microelectrode-guiding cannula was inserted stereotactically. Commercially available microelectrodes (Atlantic Research System, Inc., Atlanta, GA) were used with the initial impedance measured between 0.5 and $1.2 \mathrm{Mohm}$. The microelectrode was advanced via the guiding cannula, and single unit records were sought from $20 \mathrm{~mm}$ above the tentative target to $5 \mathrm{~mm}$ below. Signals were displayed on an oscilloscope and on speakers. The units were analyzed on-line and were mapped on graph paper, taking into account their distance from the tentative target, the firing pattern and 
frequency, and the response to movement-related activity. Differentiation of neurons was accomplished according to established criteria.[16,30,38,40] Commonly, neurons of the putamen, the GPe, the laminar borders, and the GPi were mapped along the pathway. When the ventral border of the GPi was identified, the recording was supplemented by microstimulation at 3 to $4 \mathrm{~mm}$ below it. When the first track was judged satisfactory, no additional tracks were made. In the majority of patients one or two pathways were investigated, but in individual patients three to five pathways were explored.

A 1.1-mm unipolar electrode with a 3-mm uninsulated tip was used for macrostimulation and lesion making. The tip of the electrode was placed within $1 \mathrm{~mm}$ of the ventral border of the GPi according to the mapping. Macrostimulation was used to assess the threshold for the spread of current to the optic tract and the internal capsule using frequencies ranging from 5 to $100 \mathrm{~Hz}$ at 0 to $4 \mathrm{~V}$. This location was considered the final target: in case no optic or capsular responses were elicited below $2 \mathrm{~V}$. Otherwise the electrode was withdrawn in $0.5-\mathrm{mm}$ increments and the stimulation was repeated. Two or three lesions spaced $3 \mathrm{~mm}$ apart were made with the electrode tip along the same trajectory by heating the electrode with the temperature controlled at $75 \check{\mathrm{s} C}$ for 60 seconds. In some of the initial patients in the series the most cranial lesion was made at 4 to $5 \mathrm{~mm}$ above the first lesion. During lesioning the strength and mobility of the contralateral arm, speech, and visual fields were monitored. Total operative time ranged between 3 and 5 hours.

\section{Magnetic Resonance Imaging Investigation and Evaluation}

Patients underwent sequential MR imaging according to a standard preoperative protocol, in the early postoperative period (within 1 and 3 days), and at a subsequent follow-up examination (generally at 6 months, [9 months in five patients]). Magnetic resonance studies were performed with a 1.5 tesla magnet (Signa; General Electric Medical Systems). Sequences consisted of multiplanar $\mathrm{T}_{2}$-weighted fast spin-echo (FSE) sequences (TR 3083-5816 msec, TE 85-95 msec), $\mathrm{T}_{1}$-weighted FSE sequences (TR 500-700 msec, TE $11 \mathrm{msec}$ ), and proton-density weighted sequences (TR 2083-2200 msec, TE $34 \mathrm{msec}$ ). Slice thickness was 3 to $5 \mathrm{~mm}$ in different sequences, with interslice gaps of 1 to $1.5 \mathrm{~mm}$. Additional sequences included axial 3 to $5 \mathrm{~mm} \mathrm{~T}_{1}$-weighted inversion recovery images and 1-mm fast-spoiled GRASS (FSPGR) images. The field of view was $24 \mathrm{~cm}$. The MR images were analyzed independently by two investigators.

The early-phase MR images were examined for lesion size and location, the presence of additional perilesional edema, and other postoperative sequelae. The three concentric zones of the elliptic radiofrequency lesion were defined according to the criteria of Tomlinson, et al:[41] a hyperintense inner zone; a hypointense middle zone; and a hyperintense outer zone on the $\mathrm{T}_{2}$-weighted MR images. Three diameters were determined for each lesion zone: the long axis along the electrode track as well as its perpendicular were measured on sagittal images and the mediolateral diameter was measured on axial images. Total lesion zone volumes were calculated with the formula $V=4 / 31 / 2 * r_{1} * r_{2} * r_{3}$.

The location of the lesion was also evaluated using the other MR sequences considering four distinct anatomical domains: the GPi, the GPe, the putamen, and the internal capsule. It was determined whether each of these domains harbored the total lesion, the major part of the lesion, approximately half of the lesion, a minor portion of the lesion, or whether it was unaffected by the lesion. The border between the GPi and GPe was often difficult to identify. Only the "total lesion," as defined by the outer zone, was considered for this rating scale. Initially we also tried to determine the location of the middle zone only. 
This procedure, however, was abandoned because of its high interrater variability. The proximity of the outer lesion zone to the optic tract or the choroidal fissure was measured on coronal and sagittal $\mathrm{T}_{2}$-weighted $\mathrm{MR}$ images. We also investigated the distribution of additional edema surrounding the three-zoned lesion and determined whether, and to what extent, it involved the GPi, the GPe, the putamen, and the internal capsule. Edema affecting these structures was rated as not present, minor, or major.

The volume of the late-phase lesion was also determined using $\mathrm{T}_{2}$-weighted $\mathrm{MR}$ images. Only one core zone, which had a hyperintense appearance, was identified consistently. Occasionally, this hyperintense area was surrounded by small hypointense margins; however, in general, it was not possible to differentiate this area from the GPi itself, which is also hypointense on $\mathrm{T}_{2}$-weighted images. The volume of the core lesion and its location were determined as described previously.

\section{Statistical Analysis}

To allow comparative and correlative analyses between the degree of clinical improvement at the 6-month follow-up examination and the MR findings, individual indices for the relative change of the scores of the ordinal scales were calculated. The improvement index for an outcome measure was obtained by dividing the delta between the preoperative and the postoperative score by the preoperative score. This procedure yielded figures ranging between 0 and 1, wherein 0 indicated that no benefit was achieved, and 1 indicated the maximum (hypothetical) clinical benefit. For statistical analyses the Student t-test, the Wilcoxon signed-rank test, the Pearson product moment correlation, the Spearman rank-order correlation, the chi-square test, and Fisher's exact test were used. Computations were performed using a comercially available software package (Sigmastat; Jandel Scientific, San Rafael, CA).

\section{RESULTS}

\section{Clinical Features and Outcome}

Twenty-seven patients underwent left-sided and nine patients underwent right-sided pallidotomy. In two patients the operation was discontinued before a lesion was made, but completed successfully in a second session. The surgery was discontinued because of technical reasons in one patient, and the other patient had suffered a vasovagal syncope.

Details of clinical outcome will be published elsewhere. Parkinsonian symptoms and levodopa-induced dyskinesias were improved in all patients in the early postoperative period. Five patients (14\%) experienced transient side effects. One patient had mild Broca's aphasia that was found to be secondary to a small ischemic infarction involving the corona radiata, the periventricular white matter, and the dorsal thalamus. Four patients displayed an altered mental status consisting of varying degrees of disorientation, confusion, agitation, or sleepiness. One of these patients complained about altered color perception, as if seeing his environment through a greenish film. All side effects completely resolved spontaneously, usually before discharge from the hospital. 


\section{TABLE 1}

CLINICAL OUTCOME IN 36 PATIENTS WHO UNDERWENT UN LATERAL PALLIDOTOMN: COMPARISON BETWEEN PREOPERAT YE AND 6-MONTH FOLLOW-UP UPDRS SUBSCORE ${ }^{\star}$

\begin{tabular}{|c|c|c|c|c|}
\hline Factor & Preop Score & $\begin{array}{l}\text { 6+Mo Follow- } \\
\text { Up Score }\end{array}$ & $\begin{array}{l}\text { Relative } \\
\text { Change }\end{array}$ & p Value \\
\hline $\begin{array}{l}\text { motor soore "off" } \\
\text { UPDRS }\end{array}$ & $58.1 \pm 13.8$ & $33.0 \pm 9.8$ & 0.43 & $<0.0001$ \\
\hline $\begin{array}{l}\text { ADL soore "off" } \\
\text { UPDRS }\end{array}$ & $31.4 \pm 6.1$ & $18.2 \pm 4.6$ & 0.42 & $<0.0001$ \\
\hline $\begin{array}{l}\text { trad ykinesia score "off' } \\
\text { contralateral extrem ities } \\
\text { UPDRS (scores } 23-26 \text { ) }\end{array}$ & $11.6 \pm 2.3$ & $5.6 \pm 2.5$ & 0.52 & $<0.0001$ \\
\hline $\begin{array}{l}\text { percentage of anake time } \\
\text { w/dyskinesias }\end{array}$ & $37.5 \pm 21.4$ & $18.1 \pm 15.7$ & 0.52 & $<0.0001$ \\
\hline
\end{tabular}

The global outcome at 6-month follow-up examination was rated by the evaluating neurologist as markedly improved in 22 patients (61\%), moderately improved in 12 patients (33\%), and only slightly improved in two patients $(6 \%)$. The latter two patients themselves rated their condition as unchanged. The pre- and postoperative mean values for the UPDRS measures used in this analysis and the corresponding improvement indices are given in Table 1 . The changes were highly significant for all outcome measures. Dyskinesias contralateral to the side of the surgery were abolished or almost abolished in 25 of the 33 patients who had preoperative dyskinesias (76\%) and moderately or markedly improved in seven patients $(21 \%)$. In one patient, a 49-year-old woman, dyskinesias were similar to their preoperative appearance.
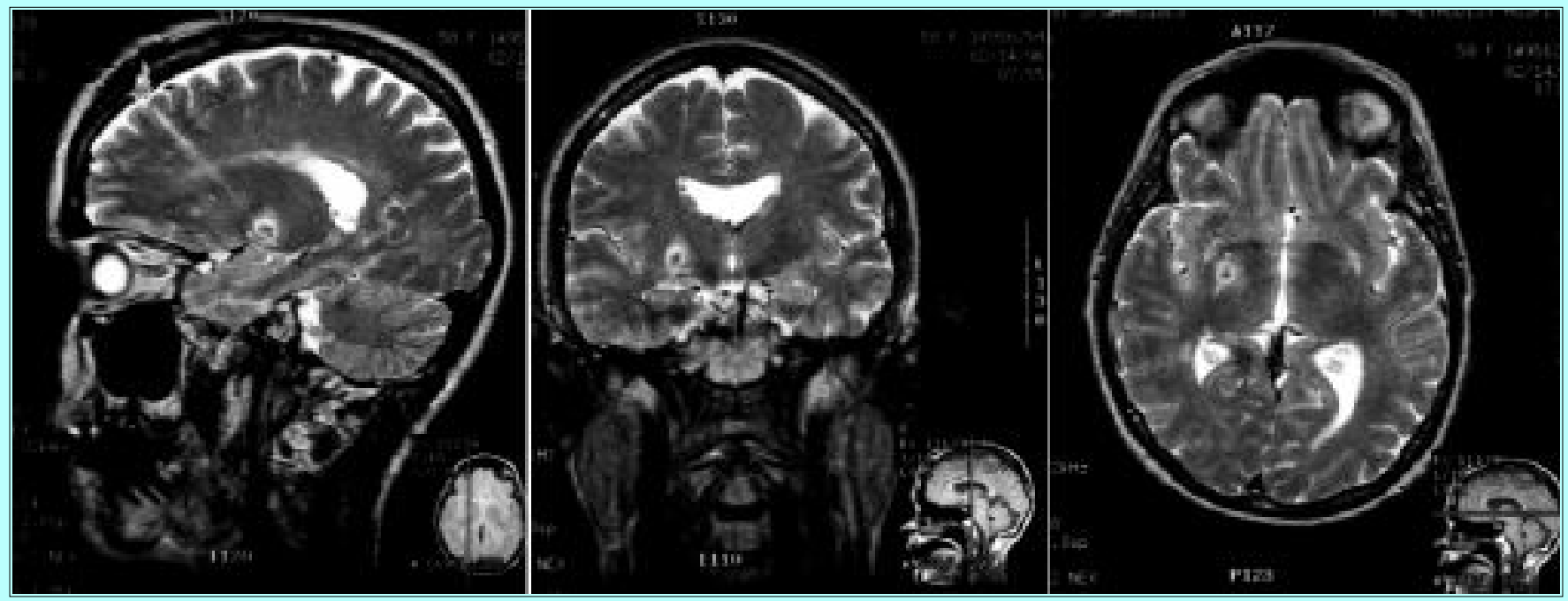

Fig. 1. Magnetic resonance $\mathrm{T}_{2}$-weighted sequences obtained in a 58-year-old patient 2 days after a right-sided pallidotomy was performed. The sagittal (left), coronal (center), and axial (right) images demonstrate the elliptoid shape of the lesion and its three-zoned composition with a dotlike inner hyperintense zone, a hypointense middle zone, and a hyperintense outer zone.

\section{Magnetic Resonance Imaging Evaluation of Early-Phase Lesions}

Early MR examinations performed at a mean of 2.1 days were available in 34 of the 36 patients. The 
lesions commonly had the shape of a prolate spheroid, with the long axis along the electrode trajectory (Fig. 1). The three-zoned composition of the lesion was seen on all imaging sequences (Fig. 2).

Demarcation between the different zones was best seen on the $\mathrm{T}_{2}$-weighted $\mathrm{MR}$ images. The inner zone had a dot like appearance on the axial images and a cylindrical shape on the sagittal images. The diameter of this zone did not exceed $1 \mathrm{~mm}$ on the axial views. The mean volume of the middle zone of the lesion was $44.4+/-17.6 \mathrm{~mm}^{3}$ (range $14-92 ; \mathrm{P}_{25}=33, \mathrm{P}_{75}=52$ ), and the mean lesion volume, as defined by the outer zone, was $262.2+/-111.6 \mathrm{~mm}^{3}$ (range 65-576; $\mathrm{P}_{25}=189, \mathrm{P}_{75}=335$ ). There was a highly significant positive correlation between the volume of the middle zone and the total lesion volume $(\mathrm{r}=0.75, \mathrm{p}<0.0001)$.
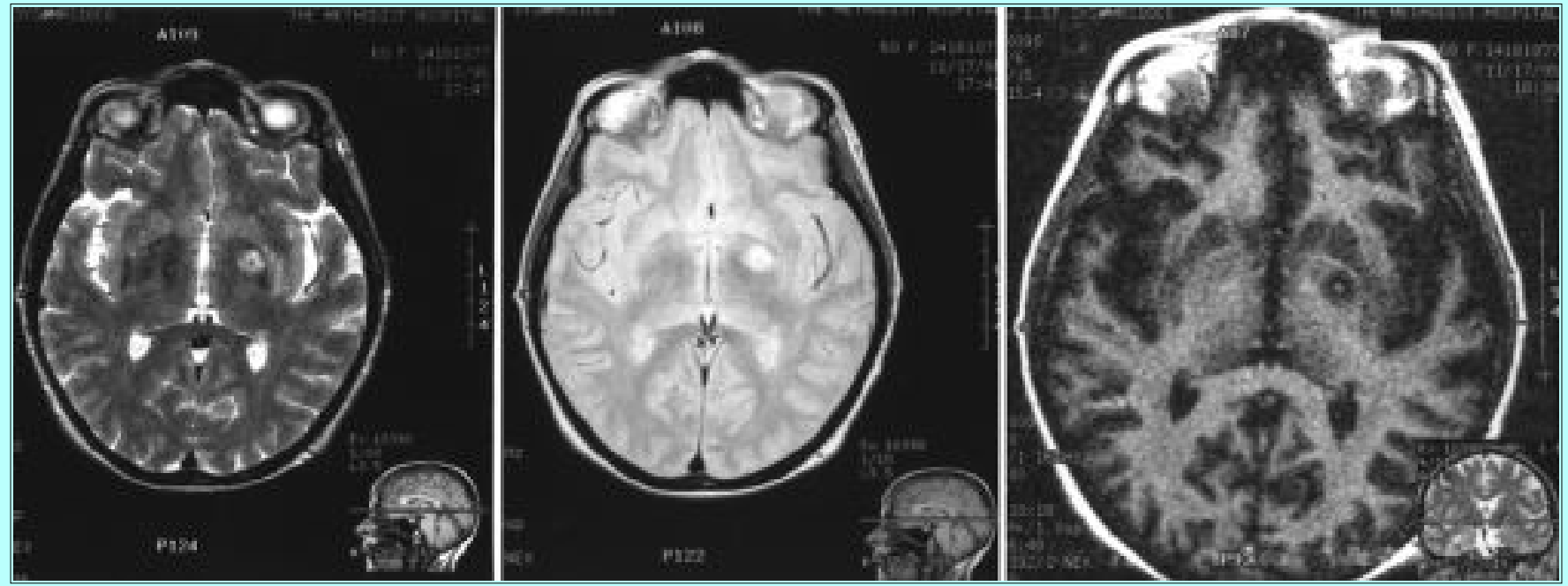

Fig. 2. Magnetic resonance images comparing different sequences obtained in a 60-year-old patient 1 day after a left-sided pallidotomy was performed. The three-zoned composition of the lesion is seen on axial $\mathrm{T}_{2}$-weighted images (upper), proton density-weighted images (center), and inversion recovery images (lower).

The early-phase lesion, as defined by the outer zone, involved the GPi in all cases. It was always located within the lateral portion of the GPi in immediate proximity to the posterior limb of the internal capsule. In 11 patients (32\%) the lesion was located completely within the GPi, in 16 patients (47\%) the major portion of the lesion was located in the GPi but also involved the GPe to a minor extent, in four patients (12\%) approximately half of the lesion was located in the GPi and the other half in the GPe, and in two patients $(6 \%)$ the major portion of the lesion was located in the GPi but it also encroached on the internal capsule to a minor extent. In one patient the major part of the lesion involved the GPe. Commonly, the medial margin of the lesion was located above the most lateral aspect of the optic tract or just lateral to it. The distance from the most inferior margin of the outer zone to the optic tract or the choroidal fissure on the coronal and sagittal $\mathrm{T}_{2}$-weighted images ranged between 0 and $1.5 \mathrm{~mm}$ in 27 cases $(79 \%)$ and between 2 and $5 \mathrm{~mm}$ in six cases (18\%) (Fig. 3). 


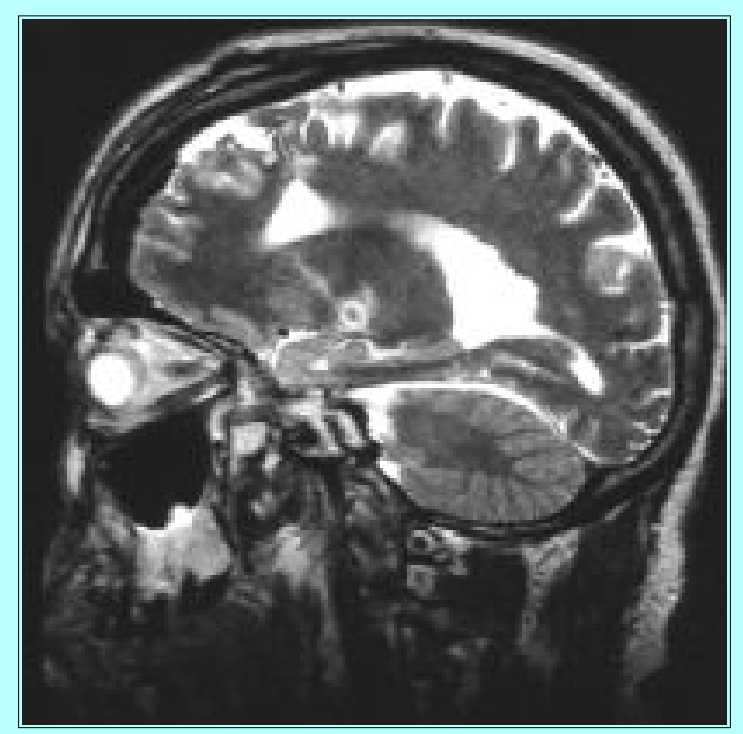

Fig. 3. Sagittal $T_{2}$-weighted MR imaging sequence in a 40 -year-old patient 2 days after a left-sided pallidotomy was performed. The image shows the proximity of the inferior margin of the hyperintense outer zone of the lesion to the optic tract.

In all patients additional edema surrounding the three-zoned lesion was detected. The GPi was involved to a major extent in 32 patients and to a minor extent in two, whereas the GPe was affected to a major extent in 20 and to a minor extent in 12 cases (Fig. 4).
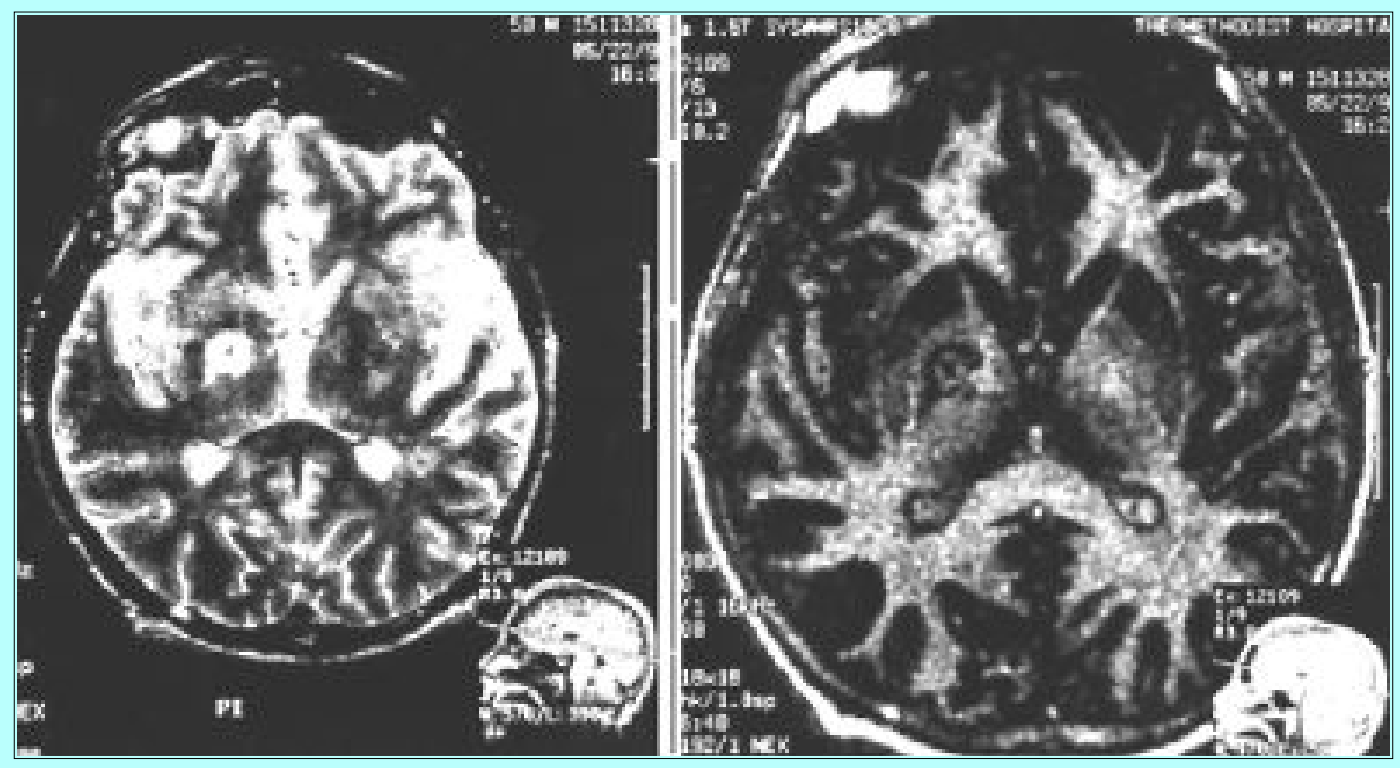

Fig. 4. Axial $T_{2}$-weighted (left) and inversion recovery (right) MR sequences in a 50-year-old patient after a right-sided pallidotomy was performed. The lesion is confined to the medial pallidum, which is best shown on the inversion recovery sequences (compare with the morphology of the contralateral pallidum). Additional perilesional edema extends into the adjacent internal capsule.

With the exception of two cases, some degree of edema was also seen in the internal capsule. Occasionally mild edema extended along the corticospinal tract fibers down to the cerebral peduncle. However, none of these patients had evidence for corticospinal tract dysfunction. Although the lesion never encroached on the optic tract, varying degrees of signal alteration of the optic tract were found in 11 cases (Fig. 5). This radiographic finding was not associated with visual field defects. It was also found 
in the patient who had had altered color perception postoperatively. Optic tract edema tended to be more frequent when lesions were located more inferiorly.
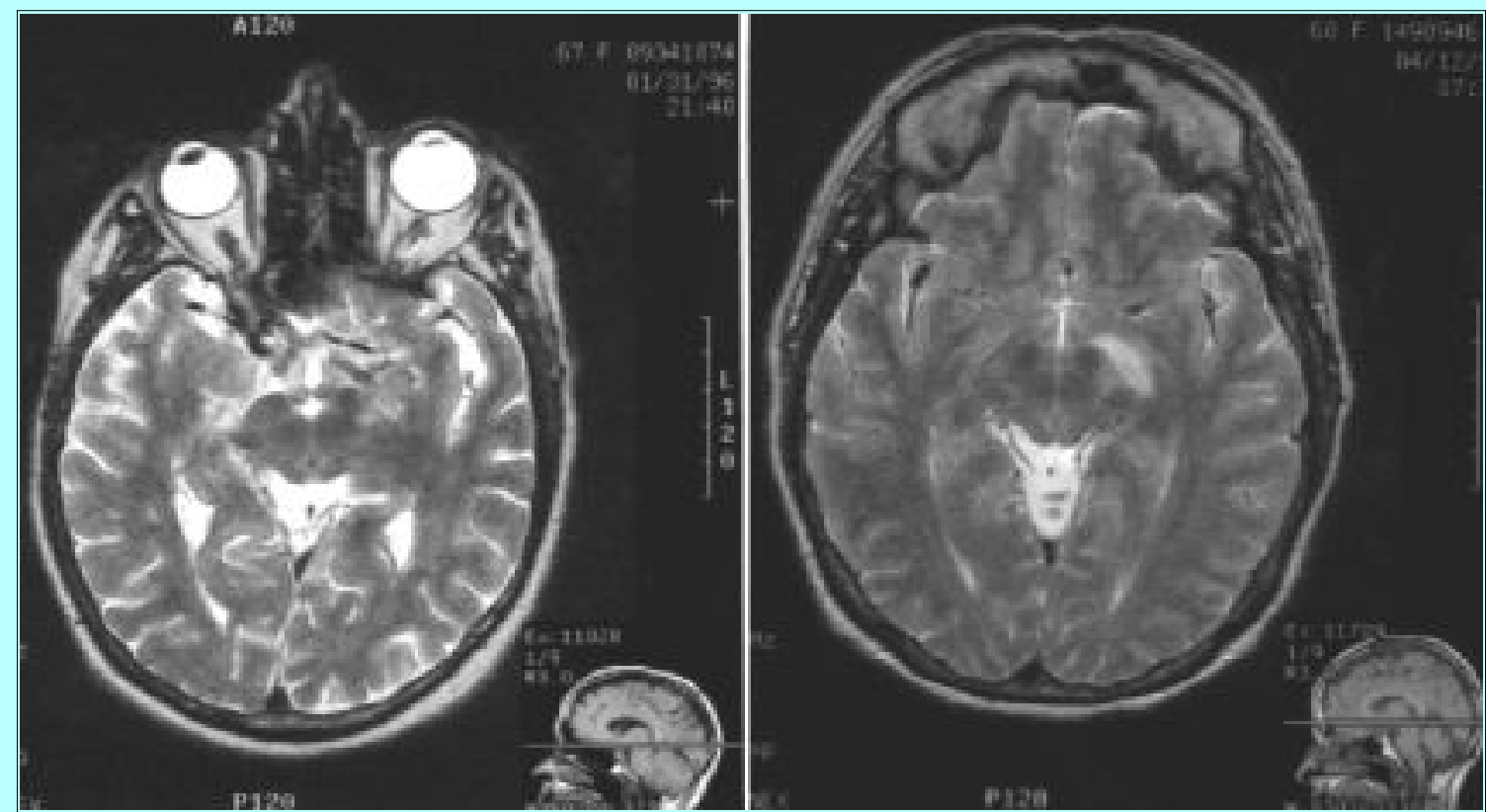

Fig. 5. Axial $\mathrm{T}_{2}$-weighted $\mathrm{MR}$ images obtained in a 67-year-old patient (left) and a 60-year-old patient (right) 2 days after left-sided pallidotomy. In both patients asymptomatic edema of the optic tract is detected.

There was no evidence of hemorrhage along the electrode trajectory or in the target area. In two cases ischemic infarctions were detected in the corona radiata and periventricular white matter. In one instance this was an incidental finding (Fig. 6), whereas the infarction resulted in moderate transient aphasia in the other case as described above. In another patient, a frontal cortical-subcortical venous infarction was detected at the entry site of the electrode. This 61-year-old woman did not experience any adverse effects postoperatively. 


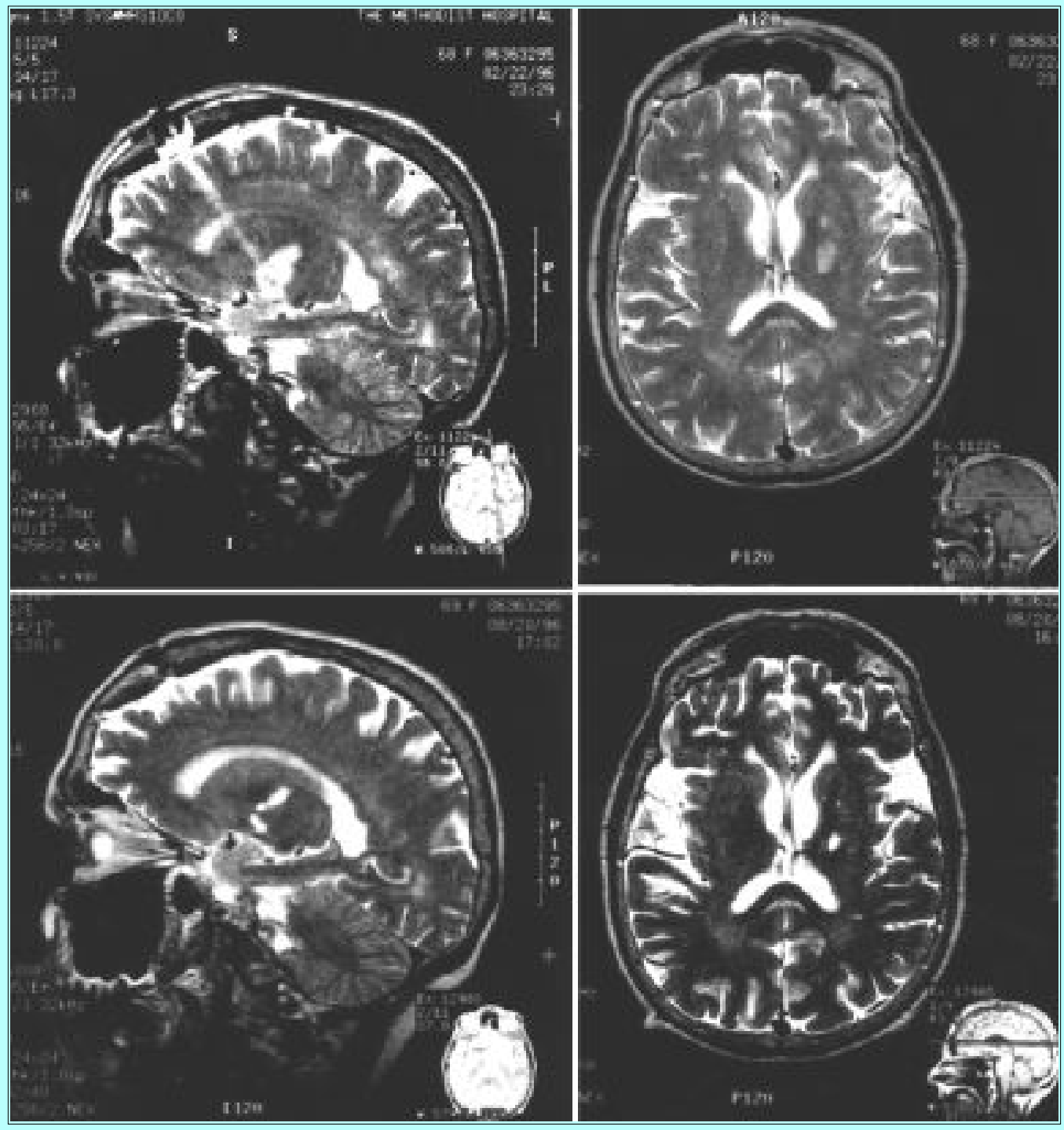

Fig. 6. Magnetic resonance $T_{2}$-weighted sequences obtained in a 68 -year-old patient 2 days (upper left and right) and 6 months (lower left and right) after a left-sided pallidotomy. The early sagittal (upper left) and axial (upper right) images show the incidental finding of an asymptomatic small ischemic infarction extending from the pallidal lesion area into the corona radiata. The lesion and the infarction are well demarcated on the sagittal (lower left) and axial (lower right) follow-up studies.

\section{Magnetic Resonance Imaging Evaluation of Late-Phase Lesions}

Late-phase MR images were available in 32 of the 36 patients. In 29 of those patients clearly discernible lesions were identified, whereas in three patients the lesions had disappeared. Similarly as seen in the early-phase lesions, the longitudinal axis of the lesion was oriented along the electrode trajectory (Fig. 7). The mean volume of late-phase lesions was $22+/-28.8 \mathrm{~mm}^{3}$ (range 0.5-94; $\mathrm{P}_{25}=1, \mathrm{P}_{75}=28$ ). 

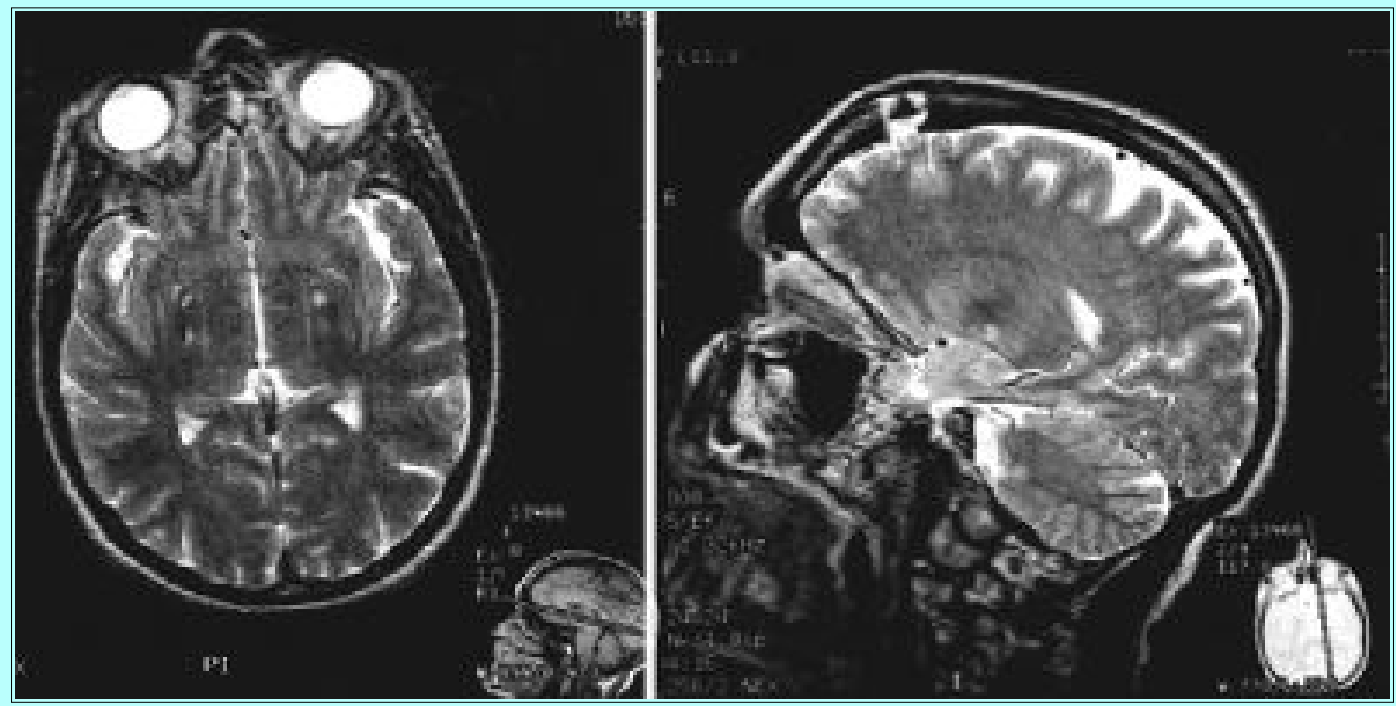

Fig. 7. Magnetic resonance $T_{2}$-weighted sequences obtained in a 51-year-old patient 6 months after a left-sided pallidotomy was performed. The axial (left) and sagittal (right) images show the hyperintense late-phase lesion and its proximity to the optic tract and choroidal fissure.

The hyperintense lesion was located in the GPi in 28 of the 29 patients in whom a lesion was identified (Fig. 8). It was completely located within the GPi in 23 patients (79\%), it was located mainly in the GPi but also involved the GPe in two patients (7\%), and it extended slightly into the internal capsule in three patients $(10 \%)$. In the patient in whom the major portion of the early-phase lesion had involved the GPe, the late-phase lesion was confined to the GPe (Fig. 9). The distance from the inferior border of the lesion to the optic tract or the choroidal fissure ranged from 0.5 to $2 \mathrm{~mm}$ in 22 patients (76\%) and from 3 to 5 $\mathrm{mm}$ in six patients $(21 \%)$. Edema of the optic tract had disappeared in all cases. Small demarcated residual lesions were found in those two patients who had had early ischemic infarctions (Fig. 6).

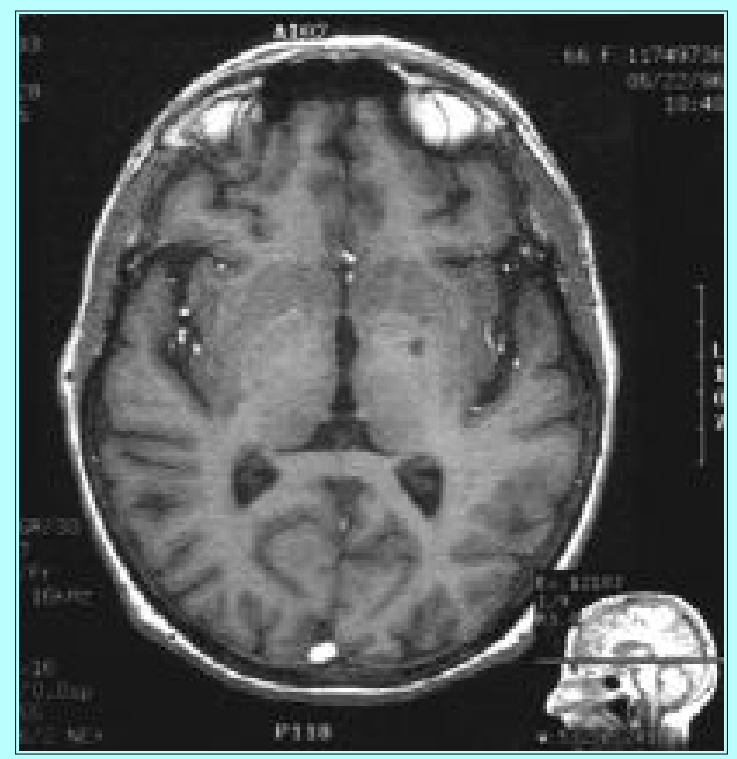

Fig. 8. Axial FSPGR MR sequence obtained in a 66-year-old patient 6 months after a left-sided pallidotomy was performed. The pallidal lesion is well demarcated.

\section{Correlation Between Size of Early-and Late-Phase Lesions}

There were no significant relationships between the volumes of the middle zone of the early-phase lesion 
and the volumes of the late-phase lesion. There was also no relationship between the size of the total early-phase lesion and the size of the late-phase lesion. The volumes of the middle zone of the early-phase lesions in those three patients, in whom no lesion was detectable on the late-phase studies, were $28 \mathrm{~mm}^{3}, 52 \mathrm{~mm}^{3}$, and $73 \mathrm{~mm}^{3}$, respectively.

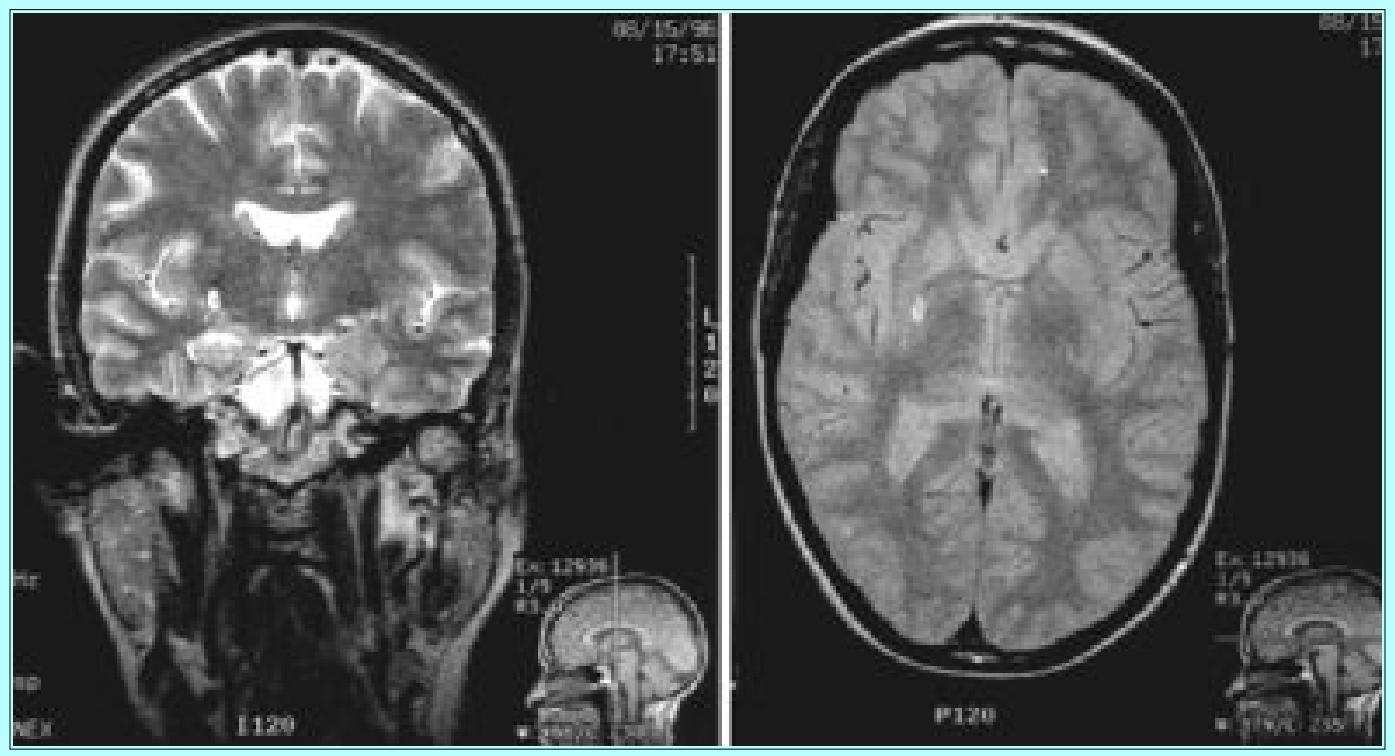

Fig. 9. Magnetic resonance $\mathrm{T}_{2}$-weighted coronal (left) and proton-density weighted axial (right) sequences obtained in a 49-year-old patient 6 months after a right-sided pallidotomy. No persistent clinical benefit was achieved in this patient, with the late-phase lesion located completely within the lateral pallidum.

\section{Correlation of Clinical Outcome With Lesion Size and Location}

As noted previously, two patients in this series experienced only limited benefit after pallidotomy. The first patient was a 70-year-old man with an akinetic-rigid parkinsonian syndrome. Early-phase MR studies after a left-sided pallidotomy demonstrated a lesion confined to the posteroventral GPi (middle zone $29 \mathrm{~mm}^{3}$, outer zone $220 \mathrm{~mm}^{3}$, approximately $1 \mathrm{~mm}$ above the optic tract). He was mildly confused after surgery. As the course of his illness progressed, his response to levodopa became more unpredictable, and he had prolonged and more severe "off" periods with marked akinesia and increased oromandibular dystonia. The late-phase MR studies showed a $84 \mathrm{~mm}^{3}$ lesion located in the lateral posteroventral GPi. It is possible that this patient's syndrome may be evolving into another parkinsonian syndrome such as striatonigral degeneration because of his progressive axial akinetic symptoms. The second patient who did not enjoy prolonged benefit after the pallidotomy is the 49-year-old woman in whom, as noted above, the late-phase lesion was confined to the GPe. In the immediate postoperative phase there was marked improvement of her contralateral bradykinesia and levodopa-induced dyskinesias of the leg. Within 3 months, however, her symptoms had recurred. These two patients were excluded from the following correlational and contingent table analysis.

When patients with a global outcome of 4 (markedly improved) were compared with those with a global outcome of 3 (moderately improved), no significant differences were found either between the volumes of the middle and the outer zone of the early-phase lesions or with the volumes of the late-phase lesions. There were no differences between these two groups with regard to whether the lesion involved the GPi only or the GPi and the GPe or with regard to the distance of the lesion from the optic tract. 
The indices for the UPDRS outcome measures of those three patients in whom no lesion was identified on the late-phase MR studies did not differ significantly from the other patients. Statistical analyses did not detect any significant associations between the indices for the UPDRS outcome measures and the volumes of the early- and late-phase lesions, the location of the lesion (GPi only vs GPi/GPe), or the distance from the lesion to the optic tract. Contralateral dyskinesias tended to be abolished more often or almost abolished when the distance to the optic tract was less than $2 \mathrm{~mm}(\mathrm{p}=0.11)$.

No differences in lesion size and location were observed in those four patients who demonstrated transient postoperative altered mental states.

\section{DISCUSSION}

The success of functional stereotactic procedures for the treatment of movement disorders depends on a variety of factors, including patient selection, the choice of the target, the method of target localization and lesioning, the size and location of the lesion, and the experience and intraoperative judgment of the neurosurgeon. It is evident that clinical outcome is related to an interaction among these and other variables.

The clinical improvement, rated as moderate to marked in $94 \%$ of our patients 6 months postoperatively, is consistent with the results of previous series.[2,10,32] None of our patients experienced persistent side effects. Microelectrode recording was helpful in several aspects. 1) It allowed identification and delineation of the different structures along the pathway of the electrode according to their firing patterns. 2) It confirmed that the tentative target indeed corresponded to the GPi. 3) It allowed identification of the ventral border of the GPi guiding the precise location of the lesion within $1 \mathrm{~mm}$ of this border. The postoperative MR imaging findings demonstrated relatively little variation in the location of the core of the lesion. Although the extent of the lesion varied both in the early- and late-phase examinations in different patients, the lesion consistently involved the lateral portion of the posteroventral GPi except in one case. The small range of variation in clinical outcomes as well as the location of the core of the lesion most likely explains the lack of significant correlations between clinical outcome and both lesion size and location. On the other hand, our study indicates that if the lesion is precisely located at a "strategic" point, its size may not be of primary relevance to achieve good clinical improvement. Three patients in whom the acute lesion "disappeared" within 6 months had similar improvement compared with those who had a larger persisting lesion.

Limited information is available on the radiological features of stereotactic pallidal lesions. Hariz[13] reported that the mean volume of the pallidal lesion in five of his patients was $67 \mathrm{~mm}^{3}$ according to CT studies obtained 3 to 12 months postoperatively. Size and site of the lesion did not correlate with clinical outcome in this small series. The average lesion size at 1 to 3 days postoperatively according to MR studies was $127+/-35 \mathrm{~mm}^{3}$ in the series by Baron, et al.,[2] and volumes in MR studies performed within 1 week after surgery ranged between 80 to $150 \mathrm{~mm}^{3}$ in the series by Lozano, et al.[30] Lehmann, et al.,[29] contended that lesion volume was a factor affecting clinical outcome according to MR studies obtained within 1 week and 4 to 6 weeks after surgery in six patients, but no data supporting such correlations were provided.

The MR characteristics of the early- and late-phase pallidal lesions in the present study were similar to those described previously for thalamic lesions.[41] Radiofrequency lesioning has been shown to result in a hemorrhagic coagulation necrosis. $[9,41]$ The MR appearance of the different concentric zones in the 
early-phase lesions is related to specific forms of hemoglobin and to the cellular and interstitial water content. The middle zone, which is hypointense on $\mathrm{T}_{2}$-weighted MR images, is thought to correspond to the area of the hemorrhagic coagulation necrosis, and the outer hyperintense zone is thought to present perilesional edema. In our study the degree of the perilesional edema zone correlated with the volume of the middle zone. The extension of this zone is also related to other lesioning parameters such as temperature and lesion time. Our MR imaging analysis also detected additional edema, which was not concentric and less hyperintense than the outer zone of the lesion, affecting the anatomical target and adjacent structures as well. This additional edema spread along the longitudinal direction of the axons in the internal capsule and the optic tract in some patients. Such additional edema did not result in morbidity in the present series except in one patient who complained of altered color perception for a period of 4 weeks. As demonstrated previously, in general, the radiofrequency lesions shrank over time,[41] although in four patients the late-phase lesion was larger than the hypointense zone of the early-phase lesion. It has been assumed that "healing and repair" of the lesion occurs over a period of 4 months, which implies that the lesion may subsequently have a more static appearance.[41] The hyperintense late-phase lesion most probably indicates permanent tissue necrosis or gliosis. An exponential rate for the shrinkage has been postulated. With respect to the lack of correlation between the size of the early- and late-phase lesions in our study, the kinetics of the shrinkage might differ in individual patients.

The mechanisms by which pallidotomy improves parkinsonian motor symptoms and levodopa-induced dyskinesias in PD are not clear. Recent studies have provided better understanding of the functional organization of the basal ganglia circuitry and the pathophysiological mechanisms of PD. [1,3,4,6,7] In summary, the loss of dopaminergic nigrostriatal neurons is thought to result in a dysfunction of the two parallel segregated striatopallidal pathways. In the indirect pathway, increased activity of the inhibitory striato-GPe projection reduces the firing of inhibitory GPe neurons projecting to the subthalamic nucleus (STN). This in turn results in disinhibition of the STN and increased drive of the GPi via the excitatory STN-GPi projection. In particular, the development of bradykinesia is thought to be associated with excessive excitatory activity of the STN-GPi projection. The inhibitory direct striato-GPi pathway has been described as underactive in PD. Finally, the dysfunction of the striatopallidal pathways results in increased inhibitory activity and overinhibition of the ventrolateral thalamus via the GPi-thalamic projections that transverse the internal capsule in the ansa and the fasciculus lenticularis. Analagous to animal studies, the irregular high-frequency firing pattern of the GPi has often been interpreted as a correlate of pallidal hyperactivity, although so far only very limited data are available in nonparkinsonian patients. The effect of pallidotomy is thought to result mainly from reduction of pallidal overinhibition of the thalamus.

There has been considerable debate as to where the "ideal" pallidal lesion should be placed.[17,21,26,31] Should the lesion be directed primarily to abolish the neuronal hyperactivity of the GPi or the GPe, or should it interrupt pallidal outflow?

The choice of the target currently favored by most neurosurgeons was originally established by Leksell who had observed better results with posteroventral pallidotomy.[39] Leksell's methods, however, were quite different from the approaches that are used today. He performed pallidotomy using two parallel electrodes that were set simultaneously into the pallidum, $8 \mathrm{~mm}$ apart, the tip of the first electrode being located in the posteroventral GPi and the tip of the second in the posteroventral GPe. The position of these electrodes is well demonstrated in the article by Svennilson and colleagues.[39] The lesion was 
created via bipolar coagulation of the tissue between the two electrodes, which had noninsulated tips that were $10 \mathrm{~mm}$ in length. Using this method, large lesions involving both the GPe and the GPi were created. When Laitinen, et al.,[27] reintroduced Leksell's approach in the late 1980s, they used a single monopolar electrode and the lateral coordinate represented a mean value of the two coordinates used by Leksell. Interestingly, Laitinen[26] has recently contended that he intends to place the lesion in the GPe and has moved the x coordinate further laterally. The majority of neurosurgeons, however, perform posteroventral pallidotomy by lesioning the lateral GPi.[2,10,32] The coordinates that are used by most neurosurgeons describe a target that is located 20 to $21 \mathrm{~mm}$ lateral to the midline, 4 to $5 \mathrm{~mm}$ below the intercommissural line, and 2 to $3 \mathrm{~mm}$ anterior to the midcommissural point. Because of the length of the uninsulated tip of the electrodes currently used, however, the lesion may also involve dorsal and anterior aspects of the GPi.[30] This is even more important when the lesion is designed to involve kinesthetic somatosensory neurons, which may be scattered along the pathway of the microelectrode. The clinical outcome in our series did not differ between those patients in whom the lesion was located only in the GPi or in both the GPi and to a lesser extent the GPe. It is remarkable, however, that the only patient in whom the late-phase lesion was found to be completely confined to the GPe failed to achieve sustained improvement. The initial clinical benefit in this patient might be explained by edema affecting the GPi.

With respect to anatomical studies, a lesion in the posteroventral lateral GPi can affect fibers from both pallidothalamic outflow pathways, the ansa lenticularis, and fasciculus lenticularis.[5] Experiments in animals showed that ventral lesions in the caudal GPi produce degeneration in both projections. The proximity to the choroidal fissure indicates severance of these pathways in the majority of our patients. Similar to lesions in the subthalamic region, $[24,25,34]$ a small lesion located in this region might be as effective in improving parkinsonian symptoms as a larger lesion located more dorsally. It has been hypothesized that also lesioning the pallidal outflow pathways to the brainstem, in particular to the pedunculopontine nucleus, might render a beneficial effect, especially for gait disturbance.[18] We suggest that an additional aspect might be taken into account. Subpallidal lesions might also involve pallidal inflow, specifically the overactive excitatory STN-GPi pathway. Little is known about the detailed anatomy and its variations in humans. Theoretically, lesioning of the tightly packed "motor" fibers might be more beneficial than lesioning of neurons distributed over a wider area. However, selective fasciculoansatomy or subpallidotomy would be difficult to perform with current techniques. Eventually, "subpallidotomy" might be an approach that could combine both the possible advantages of targeting the STN[12] and pallidotomy in patients with PD, but obviate the risk of hemiballism. Although STN lesions may result in persistent disabling hemiballism, such hyperkinesias can be abolished by targeting the GPi.[25]

Although few patients in our study had transient postoperative side effects and no patient suffered from persistent morbidity, the postoperative MR studies revealed small ischemic and venous infarctions in three patients. These findings were asymptomatic in two cases; however, they indicate the potential risk of the procedure. Hemorrhagic complications appear to be rare with contemporary techniques. The small infarctions in the corona radiata seen in two of our patients are most probably related to ischemia in the vascular territory of perforating arteries that traverse the pallidum. It is conceivable that radiofrequency lesioning resulted in occlusion of such a small artery.

Radiofrequency lesioning is currently used almost exclusively to create therapeutic lesions in functional stereotactic neurosurgery. It has replaced various other methods such as the local injection of alcohol or chemicals, the inflation of balloons, the use of leukotomes, cooling of the probe, and the interstitial implantation of radionucleides.[14,33,36] Radiofrequency lesioning is regarded as superior to these 
methods because it allows better definition of the lesion by varying parameters such as the diameter of the electrode, the exposed length of the electrode tip, and modulation of the time and the temperature of the lesion making. The "predictability" of the lesion has been stressed, particularly with regard to experimental studies using egg white. $[9,14]$ The lack of correlation between the volumes of the earlyand late-phase lesions in our study, however, challenges this argument. Radiofrequency lesioning may not be the optimal method to produce therapeutic lesions in functional neurosurgery.

\section{CONCLUSIONS}

Posteroventral GPi pallidotomy is a highly effective and a safe treatment for advanced PD. Postoperative MR imaging is a suitable method for noninvasive examination of the pallidal lesion. Further investigations, including pathoanatomical studies, are needed to answer definitely the question of where the "ideal" lesion should be located.

\section{Acknowledgments}

We gratefully acknowledge Charles F. Contant for advice and review of the statistical analyses, Kenneth Schwartz and Lea Ben-Arie for their technical assistance, and Monica L. Domingue for assistance in the preparation of the manuscript.

\section{References}

1. Albin RL: The pathophysiology of chorea/ballism and parkinsonism. Park Rel Disord 1:3-11, 1995

2. Baron MS, Vitek JL, Bakay RAE, et al: Treatment of advanced Parkinson's disease by posterior GPi pallidotomy: 1-year results of a pilot study. Ann Neurol 40:355-366, 1996

3. Bergman H, Wichmann T, DeLong MR: Reversal of experimental parkinsonism by lesions of the subthalamic nucleus. Science 249:1436-1438, 1990

4. Brotchie JM, Mitchell IJ, Sambrook MA, et al: Alleviation of parkinsonism by antagonism of excitatory amino acid transmission in the medial segment of the globus pallidus in rat and primate. Mov Disord 6:133-138, 1991

5. Carpenter MB: Anatomical organization of the corpus striatum and related nuclei, in Yahr MD (ed): The Basal Ganglia. Research Publications: Association for Research in Nervous and Mental Disease, Vol 55. New York: Raven Press, 1976, pp 1-36

6. DeLong MR: Primate models of movement disorders of basal ganglia origin. TINS 13:281-285, 1990

7. DeLong MR, Crutcher MD, Georgopoulos AP: Primate globus pallidus and subthalamic nucleus: functional organization. J Neurophysiol 53:530-543, 1985

8. De Salles AA, Brekhus SD, De Souza EC, et al: Early postoperative appearance of radiofrequency lesions on magnetic resonance imaging. Neurosurgery 36:932-937, 1995

9. Dieckmann G, Gabriel E, Hassler R: Size, form and structural peculiarities of experimental brain lesions obtained by thermocontrolled radiofrequency. Confin Neurol 26:134-142, 1965

10. Dogali M, Fazzini E, Kolodny E, et al: Stereotactic ventral pallidotomy for Parkinson's disease. 
Neurology 45:753-761, 1995

11. Favre J, Taha JM, Nguyen TT, et al: Pallidotomy: a survey of current practice in North America. Neurosurgery 39:883-892, 1996

12. Guridi J, Luquin MR, Herrero MT, et al: The subthalamic nucleus: a possible target for stereotaxic surgery in Parkinson's disease. Mov Disord 8:421-429, 1993

13. Hariz MI: Correlation between clinical outcome and size and site of the lesion in computed tomography guided thalamotomy and pallidotomy. Stereotact Funct Neurosurg 54/55:172-185, 1990

14. Hassler R, Mundinger F, Riechert T: Stereotaxis in Parkinson Syndrome. Berlin: Springer-Verlag, 1979

15. Hassler R, Riechert T: Indikationen und Lokalisationsmethode der gezielten Hirnoperationen. Nervenarzt 25:441-447, 1954

16. Hutchison WD, Lozano AM, Davis KD, et al: Differential neuronal activity in segments of globus pallidus in Parkinson's disease patients. Neuroreport 5:1533-1537, 1994

17. Iacono RP: Stereotactic pallidotomy. J Neurosurg 85:987-988, 1996 (Letter)

18. Iacono RP, Shima F, Lonser RR, et al: The results, indications, and physiology of posteroventral pallidotomy for patients with Parkinson's disease. Neurosurgery 36:1118-1127, 1995

19. Jankovic J, McDermott M, Carter J, et al: Variable expression of Parkinson's disease: a base-line analysis of the DATATOP cohort. Neurology 40:1529-1534, 1990

20. Kelly PJ: Pallidotomy in Parkinson's disease. Neurosurgery 36:1154-1157, 1995

21. Kondziolka D, Lunsford LD: Stereotactic pallidotomy. J Neurosurg 85:986-987, 1996 (Letter)

22. Krauss JK, Akeyson EW, Giam P, et al: Propofol-induced dyskinesias in Parkinson's disease. Anesth Analg 83:420-422, 1996

23. Krauss JK, Jankovic J: Surgical treatment of Parkinson's disease. Am Fam Physician 54:1621-1629, 1996

24. Krauss JK, Mohadjer M, Nobbe F, et al: The treatment of posttraumatic tremor by stereotactic surgery. Symptomatic and functional outcome in a series of 35 patients. J Neurosurg 80:810-819, 1994

25. Krauss JK, Mundinger F: Functional stereotactic surgery for hemiballism. J Neurosurg 85:278-286, 1996

26. Laitinen LV: Pallidotomy for Parkinson's disease. Neurosurg Clin North Am 6:105-112, 1995

27. Laitinen LV, Bergenheim AT, Hariz MI: Leksell's posteroventral pallidotomy in the treatment of Parkinson's disease. J Neurosurg 76:53-61, 1992

28. Langston JW, Widner H, Goetz CG, et al: Core Assessment Program for Intracerebral Transplantations (CAPIT). Mov Disord 7:2-13, 1992

29. Lehman RM, Mezrich R, Sage J, et al: Peri- and postoperative magnetic resonance imaging 
localization of pallidotomy. Stereotact Funct Neurosurg 62:61-70, 1994

30. Lozano A, Hutchison W, Kiss Z, et al: Methods for microelectrode-guided posteroventral pallidotomy. J Neurosurg 84:194-202, 1996

31. Lozano A, Hutchison W, Kiss Z, et al: Stereotactic pallidotomy. J Neurosurg 85:988-990, 1996 (Letter)

32. Lozano AM, Lang AE, Galvez-Jimenez N, et al: Effect of GPi pallidotomy on motor function in Parkinson's disease. Lancet 346:1383-1387, 1995

33. Meyers R: The surgery of the hyperkinetic disorders, in Vinken PJ, Bruyn GW (eds): Diseases of the Basal Ganglia. Handbook of Clinical Neurology, Vol 6. Amsterdam: North-Holland, 1968, pp 844-878

34. Mundinger F: Stereotaxic interventions on the zona incerta area for treatment of extrapyramidal motor disturbances and their results. Confin Neurol 26:222-230, 1965

35. Olanow CW: GPi pallidotomy--have we made a dent in Parkinson's disease? Ann Neurol 40:341-343, 1996

36. Redfern RM: History of stereotactic surgery for Parkinson's disease. Br J Neurosurg 3:271-304, 1989

37. Stacy M, Jankovic J: Differential diagnosis of Parkinson's disease and the parkinsonism plus syndromes. Neurol Clin 10:341-359, 1992

38. Sterio D, Beric A, Dogali M, et al: Neurophysiological properties of pallidal neurons in Parkinson's disease. Ann Neurol 35:586-591, 1994

39. Svennilson E, Torvik A, Lowe R, et al: Treatment of parkinsonism by stereotactic thermolesions in the pallidal region. A clinical evaluation of 81 cases. Acta Psychiatr Neurol Scand 35:358-377, 1960 40. Taha JM, Favre J, Baumann TK, et al: Characteristics and somatotopic organization of kinesthetic cells in the globus pallidus of patients with Parkinson's disease. J Neurosurg 85:1005-1012, 1996

41. Tomlinson FH, Jack CR Jr, Kelly PJ: Sequential magnetic resonance imaging following stereotactic radiofrequency ventralis lateralis thalamotomy. J Neurosurg 74:579-584, 1991

Manuscript received January 3, 1997.

Accepted in final form February 15, 1997.

The study was partly supported by grants from the National Parkinson Foundation, the Houston Area Parkinson Society, the Ted and Irma Kaplen Endowment, the T.L.L. Temple Foundation, and The Methodist Hospital.

Address reprint requests to: Joachim K. Krauss, M.D., Department of Neurosurgery, Inselspital, University of Berne, 3010 Berne, Switzerland. 